\title{
Protagonismo do acadêmico de enfermagem em projeto de vivência universitária: hidroterapia na gestação
}

\begin{abstract}
RESUMO | Objetiva-se analisar o projeto de Vivência Universitária com gestantes em uma Unidade Saúde da Família de SinopMT, destacando o incentivo ao protagonismo do acadêmico de enfermagem. Trata-se de um relato de experiência do projeto de vivência "hidroterapia na gestação", com envolvimento de graduandos, profissionais da saúde, trabalhadores da equipe, gestantes e colaboradores da comunidade acadêmica e local. Os acadêmicos puderam desenvolver um plano de trabalho, implementá-lo e avaliá-lo, aprimorando conhecimentos e aplicando-os à realidade. Por meio do projeto, reconheceram a relevância da prática de exercícios físicos na gestação; a necessidade de articular saberes, práticas e sujeitos para ações de promoção à saúde; e as contribuições em sua formação acadêmica, por meio de projetos que o integrem ao contexto dos indivíduos/comunidades. É importante o incentivo ao protagonismo acadêmico, com vistas ao preparo profissional, assentado numa perspectiva de atendimento integral. São necessárias novas ações de promoção à saúde, incluindo gestantes/acompanhantes.
\end{abstract}

Palavras-chaves: Cuidado Pré-Natal; Enfermagem; Hidroterapia.

ABSTRACT I The aim of this study is to analyze the project of University Experience with pregnant women in a Family Health Unit of Sinop-MT, highlighting the incentive to the role of the nursing student. This is an experience report of the "hydrotherapy in the gestation" experience project, involving undergraduates, health professionals, staff workers, pregnant women and employees of the academic and local community. Academics were able to develop a work plan, implement it and evaluate it, improving knowledge and applying it to the reality. Through the project they recognized the relevance of the practice of physical exercises during pregnancy; the need to articulate knowledge, practices and subjects for health promotion actions; and, the contributions in its academic formation, through projects that integrate it to the context of the individuals/communities. It is important to encourage academic leadership, with a view to professional preparation, based on a comprehensive care perspective. New health promotion actions are needed, including pregnant women/companions.

Keywords: Prenatal Health Care; Nursing; Hydrotherapy.

RESUMEN | El objetivo de este estudio es analizar el proyecto de Experiencia Universitaria con mujeres embarazadas en una Unidad de Salud Familiar de Sinop-MT, destacando el incentivo para el papel del estudiante de enfermería. Este es un informe de la experiencia del proyecto de experiencia "hidroterapia en la gestación", que involucra a estudiantes universitarios, profesionales de la salud, trabajadores del personal, mujeres embarazadas y empleados de la comunidad académica y local. Los académicos pudieron desarrollar un plan de trabajo, implementarlo y evaluarlo, mejorando el conocimiento y aplicándolo a la realidad. A través del proyecto reconocieron la relevancia de la práctica de ejercicios físicos durante el embarazo; la necesidad de articular conocimientos, prácticas y temas para acciones de promoción de la salud; y, las aportaciones en su formación académica, a través de proyectos que la integran al contexto de los individuos/comunidades. Es importante fomentar el liderazgo académico, con miras a la preparación profesional, sobre la base de una perspectiva de atención integral. Se necesitan nuevas acciones de promoción de la salud, incluidas las mujeres embarazadas/acompañantes.

Descriptores: Atención Prenatal; Enfermería; Hidroterapia.

\section{Priscilla Shirley Siniak dos Anjos Modes}

Enfermeira. Especialista em Enfermagem em Unidade de Tratamento Intensivo Neonatal pela Universidade Cuiabá- UNIC. Doutoranda do Programa de Pós-Graduação em Enfermagem da Universidade Federal de Mato Grosso. Docente do Curso de Enfermagem da Universidade Federal de Mato Grosso, campus Sinop. Coordenadora do Projeto de Extensão.

\begin{abstract}
Ana Maria Nunes da Silva
Enfermeira. Especialista em cuidado prénatal pela Universidade Federal de São Paulo -UNIFESP/UAB. Doutoranda do Programa de Pós Graduação em Enfermagem, Faculdade de Enfermagem, UFMT, campus CuiabáMT. Docente do Curso de Enfermagem da Universidade Federal de Mato Grosso, campus Sinop.
\end{abstract}

\section{Jéssica Gonçalves Holanda Moraes \\ Enfermeira. Bacharel em Enfermagem pela Universidade Federal de Mato Grosso, campus Sinop. Consultora da Empresa Juris Ambientis Consultores.}

\begin{abstract}
Pâmela Juara Mendes de Oliveira
Enfermeira. Bacharel em Enfermagem pela Universidade Federal de Mato Grosso, campus Cuiabá. Mestrado Profissional em Ciências Odontológicas Integradas pela Universidade de Cuiabá, Brasil. Professora Substituta de enfermagem na Universidade Federal de Mato Grosso, campus Sinop.
\end{abstract}

Recebido em: 03/07/2019

Aprovado em: 16/07/2019 
INTRODUÇÃO

A prática de exercícios físicos regulares em todas as fases da vida mantém e melhora a capacidade cardiorrespiratória, reduz o risco de obesidade e comorbidades associadas e promove uma maior longevidade. Já durante a gravidez, entre os benefícios à mãe e ao bebê estariam: a redução do risco de diabetes gestacional e pré-eclampsia; a melhora do condicionamento físico materno; a promoção de ganho de peso saudável na gestação; o auxílio na perda de peso após nascimento do bebê $\hat{~}^{(1)} \mathrm{e}$ a influência na via de parto, com menor probabilidade de realização de cesariana ${ }^{(2-3)}$.

Para a prática de exercícios físicos, a associação American College of Obstetricians and Gynecologists (ACOG) recomenda que a gestante argumente com o profissional de saúde no pré-natal sobre a atividade física a ser realizada, considerando o tipo, a frequência e a sua intensidade, optando por aquela que seja mais segura e atenda melhor às suas necessidades e da gestação ${ }^{(1,4)}$.

Enfermeiros, enquanto profissionais da saúde, também são responsáveis pela promoção de hábitos de vida saudáveis às gestantes, a exemplo da orientação a prática de exercícios físicos regulares, que são seguros na gestação. Para tanto, os profissionais devem obter informações, como: período gestacional, problemas de saúde, prática de exercício físico (tipo, frequência, intensidade, como se sente durante a atividade), dentre outras. Na sequência, a partir da priorização dos diagnósticos levantados, o profissional delineará os resultados esperados, executando ao final a avaliação das intervenções propostas ${ }^{(5)}$.

Dos exercícios referenciados e de segurança no período gestacional encontram-se a natação e os realizados em água ${ }^{(1-4)}$, incluindo a hidroterapia. A hidroterapia é fundamentada nos princípios de imersão e nos seus efeitos no corpo da gestante, como o empuxo e a pressão hidrostática. O corpo imerso sofre efeito do empuxo, que é uma força exercida no sentido oposto à força do peso, proporcionando leveza, menor descarga de peso articular, maior liberdade de movimentação e alívio das algias. A pressão hidrostática auxilia o bombeamento do sistema venoso e linfático da periferia em direção aos vasos centrais. Como consequência disso, há aumento do retorno venoso, do volume sistólico e do débito cardíaco, que geram efeitos no sistema de regulação da pressão arterial (PA), cujas alterações benéficas resultam no aumento de diurese e redução da pressão arterial ${ }^{(6)}$.

\section{6 \\ Enfermeiros, enquanto profissionais da saúde, também são responsáveis pela promoção de hábitos de vida saudáveis às gestantes, a exemplo da orientação a prática de exercícios físicos regulares, que são seguros na gestação.}

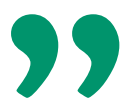

Na gestação, estudo(7) aponta alguns dos benefícios da hidroterapia, como: re- dução do nível de dor lombar, melhora no condicionamento físico, controle da frequência cardíaca da gestante e do feto, além, do registro de ganhos para a saúde mental e bem-estar físico materno.

A despeito da prática de exercícios durante a gestação, estudo( ${ }^{(8)}$ em Campinas-SP recrutou 1.279 mulheres no pós-parto, sendo constatada a caminhada como o exercício mais comumente relatado, seguido por hidroginástica. Entre os fatores positivamente associados à prática de exercício durante a gestação, foi citada a orientação profissional no pré-natal, embora, menos da metade das mulheres tivesse recebido orientação de exercícios nas consultas.

Outro estudo ${ }^{(9)}$ transversal realizado com 256 gestantes adultas no segundo trimestre gestacional, assistidas pelas unidades de atenção básica à saúde do município de Botucatu-SP, identificou que a maior parte das gestantes era insuficientemente ativa $(77,7 \%)$. Elas realizavam predominantemente atividades domésticas e/ou ocupacionais. Como consequência, a proporção de grávidas expostas aos problemas de saúde associados à prática insuficiente de atividade física na gestação era elevada, o que reforça a promoção da atividade física como uma necessária prioridade nas políticas públicas de saúde no país.

No âmbito da Atenção Básica no Brasil, conforme a Portaria n. ${ }^{\circ}$ 648/GM, de 28 de março de 2006, prevista pelo Ministério da Saúde (MS), compete a todos os profissionais, incluindo os enfermeiros, ações de promoção da saúde através de atividades orientadas aos indivíduos ou coletividades. Especificamente, a Política Nacional de Promoção da Saúde (PNPS) prevê a proposição de um cuidado que não envolve apenas as ações que operem sobre os efeitos do adoecer, mas incidam sobre as condições de vida e favoreçam a ampliação de escolhas saudáveis por parte dos sujeitos e coletividades ${ }^{(10)}$.

$\mathrm{Na}$ direção assinalada, a eleição do referencial da promoção da saúde evoca a necessidade de se criarem políti- 
cas públicas para promovê-la, uma vez que existe a necessidade do imperativo da participação social na construção do sistema e das políticas de saúde, já que perdura a impossibilidade de o setor sanitário garantir sozinho opções saudáveis para a população. Deste modo, simultaneamente, as universidades também constituem espaços sociais para a Promoção da Saúde. Elas possuem potencial para fomentar o desenvolvimento de pesquisas de inovação em tecnologias sociais que contribuirão com a saúde, aliando-se a promoção de saúde através da atuação comunitária e promoção da autonomia dos seus acadêmicos ${ }^{(11)}$.

Particularmente, no contexto da Universidade Federal de Mato Grosso (UFMT) reforça o seu importante, esta reforça o seu importante papel na formação de jovens críticos e comprometidos com a construção de uma sociedade mais justa, democrática e inclusiva e, com este propósito, fomenta, também, através da construção de projetos de Vivência Universitária o protagonismo de seus acadêmicos. Por protagonismo juvenil, apesar das diferentes interpretações, aqui a sua compreensão é traduzida como sinônimo de participação, autonomia, cidadania e responsabilidade social ${ }^{(12)}$.

Sobre os projetos de Vivência Universitária, estes são vinculados à Pró-reitoria de Cultura, Extensão e Vivência (PROCEV) e regulamentados conforme a Portaria n. ${ }^{\circ}$ 007/PROCEV/2014, a qual dispõe sobre a concessão do auxílio aos discentes matriculados na UFMT por meio de projetos de vivência universitária, concedido de forma independente ou através de parceria institucional com departamentos da UFMT. Para a participação é necessária a inscrição de um plano de trabalho pelo acadêmico, seguido da análise feita por um comitê de avaliação. Após aprovação, são destinados recursos financeiros para as despesas, conforme disponibilidade do orçamento institucional. Ao final do projeto, é feito um relatório submetido à avaliação da PROCEV ${ }^{(13)}$.

Dito isto, o presente artigo objetiva relatar a experiência de um projeto de $\mathrm{Vi}$ vência Universitária intitulado "Hidroterapia na Gestação" em uma Unidade Saúde da Família (USF) em Sinop-MT, proposto e coordenado por uma acadêmica/extensionista de enfermagem, sob supervisão da docente/coordenadora do projeto de Extensão, com atuação dos demais membros da equipe executora. Questiona-se: Como se dá a participação e o incentivo ao protagonismo acadêmico por meio de um projeto de vivência universitária? Almeja-se dar ênfase a articulação ensino-serviço-saúde e a participação acadêmica na elaboração, implementação e avaliação de um plano de trabalho de Vivência Universitária, com ênfase no cuidado à promoção da saúde de gestantes.

\section{METODOLOGIA}

Relato de experiência de um Projeto de Vivência Universitária. No ano de 2015 foi idealizado pelas docentes autoras deste texto o projeto de Extensão "Gestação: cuidados que emanam luz". Tal projeto em vigência de seu primeiro ano, contemplava a prática de educação em saúde através de rodas de conversa com gestantes e acompanhantes em uma USF de Sinop-MT. Ainda, dentro do seu conjunto de ações, se previa a realização de projetos de Vivência Universitária, sendo executada inicialmente a proposta da "hidroterapia na gestação".

A escolha pela proposta do referido projeto de vivência foi embasada na literatura científica, que comprova os benefícios desta prática no período gravídico diante das alterações corporais ocorridas, como alívio da dor lombar ${ }^{(14)}$, acrescido de que se for aplicada durante o trabalho de parto, diminui a dor ${ }^{(15)}$; a partir da concordância dos docentes membros do projeto e da equipe de saúde e; subsidiada pelas falas das gestantes nas rodas de conversas educativas, através do projeto de Extensão.

A unidade eleita para a execução do projeto de Extensão e Vivência Universitária situava-se num bairro periférico, abrangendo na estrutura física duas equi- pes de saúde. Cada equipe era composta por médico, enfermeira, técnicas de enfermagem, recepcionista e agentes comunitários de saúde (ACS).

O projeto de Vivência Universitária foi executado no mês de outubro de 2015, em 02 períodos (matutino e vespertino), com uma duração média total de 2 horas/período.

Ainda sobre a atividade, esta foi concretizada a partir da parceria com uma Instituição de Ensino Superior Privada (Universidade de Cuiabá - UNIC, campus Sinop - MT), através dos acadêmicos e docentes da Graduação em fisioterapia e apoio da Secretaria Municipal de Sinop-MT; dos médicos e enfermeiros da USF elegida; da PROCEV, através da coordenação de extensão (CODEX); das extensionistas graduandas de enfermagem e da coordenadora do projeto de Extensão, totalizando 34 pessoas.

A ausência de piscina adequada para atender às necessidades da atividade proposta na UFMT ou próxima da unidade de saúde, fez requerer a busca de um local apropriado à atividade, o que foi contemplado através da parceria com a instituição privada citada. Ainda a pedido das gestantes, houve a necessidade de contratação de um veículo para efetuar o transporte delas e dos membros do projeto ao local da atividade. Estas demonstraram o interesse em participar da atividade e verbalizaram que o acesso facilitado favoreceria a participação.

Foram oferecidas 25 vagas, tendo como público-alvo as gestantes em acompanhamento pré-natal na unidade de saúde e participantes do projeto de Extensão. A limitação das vagas visava: adequar a capacidade física da piscina; disponibilizar espaço suficiente com segurança para o transporte dos passageiros; prover a quantidade de lanches e sucos, atendendo a todos os participantes; garantir material de divulgação suficiente; assegurar a confecção da toca e top para a prática de exercício na água, em quantidade e tamanho adequados e promover um atendimento 
exclusivo e de excelência.

Para divulgação da atividade, foi elaborado um banner disposto na recepção da unidade e confeccionados convites individuais que foram entregues nas reuniões educativas do projeto de Extensão, nas consultas de pré-natal, pelos ACS e disponibilizados na unidade de saúde.

Foi solicitada a inscrição prévia das gestantes para a atividade, constando em preenchimento de um formulário e a autorização médica para a prática da hidroterapia. Na eventual não autorização médica, esta não se constituiu em um impeditivo para a participação das gestantes nas demais atividades propostas pelo projeto de Extensão. Dois meses foram destinados para divulgação da atividade e a providência da autorização médica por parte das gestantes.

\section{RELATO DA EXPERIÊNCIA}

O projeto contou com a participação de seis gestantes, sendo duas no período da manhã e quatro à tarde. Em ambos os períodos, a sequência de atividades previu o acolhimento das gestantes, as ações educativas e os exercícios em solo e água.

As participantes foram acolhidas pela equipe executora do projeto ao chegar ao local, sendo também a elas disponibilizado um lanche leve, fresco e saudável. Em espaço reservado no âmbito da unidade parceira da ação, as gestantes executaram exercícios de alongamento em solo e foram orientadas sobre a importância do parto natural e da fisioterapia na gestação, além de informações sobre o uso e aplicabilidade da massagem em bebês (shantala). Após as orientações educativas, as participantes receberam um top e uma touca elaborados pela equipe executora do projeto para a participação das atividades na água. No meio aquático as gestantes desenvolveram exercícios aeróbicos e de relaxamento sob orientação profissional. Um ambiente descontraído foi favorecido pela interação entre os participantes.

Após o término das ações, as gestantes foram conduzidas à unidade de saúde no transporte disponibilizado pela equipe executora do projeto. Importante destacar que, a instituição parceira, UNIC, se comprometeu em dar continuidade na hidroterapia às gestantes participantes durante toda a gestação.

Os acadêmicos também puderam participar das várias ações que envolveram o projeto de vivência, sob coordenação da acadêmica-extensionista e orientação e acompanhamento da docente coordenadora do projeto de Extensão. Particularmente, quanto à participação da acadêmica como coordenadora do projeto de Vivência Universitária, entre as suas ações desenvolvidas, estiveram inclusas: a elaboração do projeto, a articulação de parcerias, a definição do local e do número de vagas, a divulgação do evento, as inscrições das participantes, a previsão dos recursos necessários, a programação e a operacionalização da ação junto aos demais membros do projeto.

Na formação acadêmica, de um modo geral, oos discentes puderam vivenciar: 1 ) o planejamento, a execução e a avaliação das ações, com flexibilizações do plano de trabalho inicial; 2) o desenvolvimento de competências e habilidades, como o trabalho em equipe; 3 ) a socialização de conhecimento e a interação com os diferentes saberes, sujeitos e setores envolvidos na atividade; 4) a promoção da saúde através da prática de atividade física; 5) a oferta de serviços em saúde, não comumente disponibilizados pelo Sistema Único de Saúde (SUS); 6) a reflexão em torno de uma postura crítica e transformadora da realidade social; 7) a oportunidade de experiências e conhecimentos, a partir de uma realidade diversa daquela vivenciada em sala de aula; e 8) a expansão de seus horizontes e ideias em busca da elaboração e transmissão do conhecimento de forma significativa; dentre outros.

\section{DISCUSSÃo}

Da literatura em periódicos, os textos que destacam o protagonismo dos acadêmicos em projetos de integração univer- sidade-comunidade, voltados ao período do ciclo gravídico-puerperal, versam habitualmente sobre as práticas educativas orientadas a gestantes ou casais. A maioria deles trata de projetos na modalidade de Extensão e não de Vivência Universitária, apesar disto, seus achados corroboram com os identificados neste trabalho, ao citar como contribuições aos acadêmicos: oportunidade de interagir com a comunidade, aprimorar e trocar conhecimentos, aplicar o conteúdo teórico na prática, experienciar a responsabilidade de coordenar atividades grupais e retribuir a sociedade o investimento do ensino recebido ${ }^{(16,17)}$.

Autores $^{(16)}$ compreendem que os acadêmicos que vivenciam a experiência de práticas em grupo tornar-se-ão futuros profissionais que assistirão às gestantes com o preparo científico atrelado às experiências de cada mulher grávida e sua família e, portanto, com maior potencial de refletir, produzir e gerar mudanças a partir da problematização da realidade dos sujeitos.

Corroborando com o exposto, estu$\mathrm{do}^{(18)}$ reforça que o interesse do profissional pela gestante e suas necessidades de saúde contribuiu para a produção de mudanças concretas e saudáveis nas atitudes das mesmas e de sua família. Para tanto, ao contrário de uma abordagem individualizada e mecanizada em saúde, se requer um atendimento ampliado e integral, que considere as singularidades dos sujeitos e articule os diferentes conjuntos de ações, incluindo aqueles relativos à promoção da saúde ${ }^{(18)}$.

Para tanto, a fim de problematizar as situações do cotidiano de suas práticas de um modo ampliado, a formação exige um processo educativo que envolva os profissionais e seus saberes técnicos em concepções holísticas e requer estratégias de ensino que abranjam a complexidade da saúde, incluindo a sua promoção. Um processo de ensino dialógico promove espaços de reflexão sobre os desafios da prática profissional e fornece aos trabalhadores a possibilida- 
de de atuar como sujeitos partícipes no processo de construção do seu conhecimento, e protagonistas na reorientação das ações de saúde ${ }^{(19)}$.

Nesta direção, as universidades possuem um importante papel na educação, na construção de competências, na troca de conhecimento e na investigação e é capaz de atrair e conciliar os saberes e as práticas intra e interdisciplinares em prol do benefício da sociedade. Portanto, enquanto centros de aprendizagem e desenvolvimento, criatividade e inovação podem contribuir para a construção, divulgação e aplicação de conhecimento sobre saúde pública e promoção da saúde ${ }^{(20)}$.

As instituições de ensino superior têm a possibilidade de influenciar positivamente a saúde e a vida da comunidade acadêmica e/ou local se inves- tirem na capacitação e promoção da emancipação dos seus membros. A capacitação dos acadêmicos e docentes permitirá que os mesmos, no exercício da sua responsabilidade individual e social pela saúde, façam escolhas saudáveis no dia a dia e se comprometam na defesa da saúde global, através do exercício da cidadania ou do desempenho de papéis, com impacto nas futuras políticas de saúde ou nas tomadas de decisão sobre as mesmas ${ }^{(20)}$.

Como recurso e parte integrante da sociedade, as universidades podem fazer uso do seu conhecimento, liderança, credibilidade e meios para fomentar a consciencialização, o diálogo e a participação na mudança em diversos contextos, de modo a contribuir com a saúde ou outras demandas locais, nacionais e internacionais ${ }^{(20)}$.

\section{CONCLUSÃO}

O incentivo para o exercício da proposição do projeto de vivência possibilitou aos acadêmicos ir além da aquisição de conhecimentos técnicos, mas de um pensar e agir crítico, responsável e sensível às necessidades de saúde dos sujeitos. Mais do que a inserção em projetos de integração ensino-serviço, os acadêmicos puderam propor e coordenar uma ação, protagonizando uma atividade intersetorial no âmbito da Atenção Básica. Ainda que a vivência relatada não possa ser analisada de modo generalizado, ela colabora com a apresentação de possibilidades de articulações e estratégias vivenciadas por acadêmicos de enfermagem no SUS. Deve-se investir em pesquisas e práticas que corroboram para uma formação crítica, autônoma e comprometida com a promoção da saúde.

\section{Referências}

1. Committee on Obstetric Practice. No. 650. Exercise during pregnancy and the postpartum period. ACOG Clin Rev. [Internet]. 2015 Dec [citado $2017 \mathrm{abr}$ 12].126: 135-142. Disponivel em: http://www.acog.org/-/media/Committee-Opinions/Committee-on-Obstetric-practice/ co650. pdf?dmc=1.

2. Dumith SC, Domingues MR, Mendoza-Sassi RA, Cesar JA. Atividade física durante a gestação e associação com indicadores de saúde materno-infantil. Rev. Saúde Públ. [Internet]. 2012 [citado 2017 mar 30]; 46(2):327-333. Disponível em: http://www.scielo.br/pdf/rsp/v46n2/3380.pdf.

3. Matoso LML, Matoso MBL. Evidências científicas acerca dos exercícios físicos durante a gravidez: uma prática possivel? C\&D-Revista Eletrônica da FAINOR. 2018; 11(1):31-47.

4. American College of Obstetricians and Gynecologists. Women' health care physicians. Frequently asked Questions FAQ119. ACOG Clin Rev. [Internet]. 2016 May [citado 2017 abr 12]. Disponível em: https://www.acog.org/-/media/For-Patients/faq119.pdf?dmc=1\&ts=20161231 T1413462496.

5. Butes L, Marinho SP, Silva A, Gomes d Costa F, Ramos AL. A atividade física na gravidez: Intervenção do Enfermeiro. Percursos [Internet]. 2010 jul-set [citado $2017 \mathrm{mar}$ 30]; 17. Disponível em: https://comum.rcaap.pt/bitstream/10400.26/9211/1/Revista\%20Percursos\%20n17_A\%20actividade\%20f\%C3\%ADsica\%20na\%20gravidez\%20-\%20interven\%C3\%A7\%C3\%A30\%20 do\%20Enfermeiro.pdf.

6. Martins N, Ruckert D. A hidroterapia como recurso para o alívio da lombalgia em gestantes. Rev. Perspecti. Ci.e saúde. 2018; 3(2):02-11.

7. Barros et al. Efeito do tratamento hidroterápico na dor lombar e qualidade de vida de gestante: estudo de caso Repercussões maternas e perinatais da hidroterapia na gravidez. Colloquium Vitae. 2018; 10(1):74-79.

8. Nascimento SL, Surita FG, Godoy AC, Kasawara KT, Morais SS. Padrões de Atividade Física e Fatores Relacionados ao Exercício durante a Gravidez: Um Estudo Transversal. PLoS One [Internet]. 2015; 10(6):e0128953. Doi: 10.1371

9. Carvalhaes MABL, Martiniano ACA, Malta MB, Takito MY, Benício MHA. Atividade física em gestantes assistidas na atenção primária à saúde. Rev. Saúde Públ. 2013;47(5):958-967.

10. Ministério da Saúde (BR). Política Nacional de Promoção da Saúde. Aprovada pela Portaria n. ${ }^{\circ} 687$, de 30 de março de 2006. Brasília (DF); 2006.
11. Tavares MF et al. A promoção da saúde no ensino profissional: desafios na Saúde e a necessidade de alcançar outros setores. Ciência \& Saúde Coletiva [Internet]. 2016 [citado 2019 jul 09]; 21(6):1799-1808. Doi: https://doi. org/10.1590/1413-81232015216.07622016.

12. Silva MAS, Ximenes VM. Políticas públicas e juventude: análises sobre o protagonismo juvenil na perspectiva dos jovens pobres. Pesqui. prát. Psicossociais. 2019; 14(1):1-15.

13. Universidade Federal de Mato Grosso. Portaria n. ${ }^{\circ} 007 / P R O C E V$, de 21 de fevereiro de 2014. Dispõe sobre a concessão de auxílio vivência universitária aos discentes da UFMT para apresentação de projetos artísticos, culturais, esportivos e de vivência universitária da UFMT [Internet]. [citado 2017 abr 12]. Disponível em: https://www.google.com.br/url?sa=t\&rct=j\&q=\&esrc $=$ s\&source $=$ web \&cd $=1 \&$ ved=0ahUKEwjPiubbv6HTAhWDiJAKHZOpBaEQFggcMAA\&url=http\%3A\%2F\%2Fwww.ufmt.br\%2Fprocev\%2Farquivos\%2F151 ae4a2e490658e077a274885581d7f.doc\&usg=AFQjCNF1 fb5qy00549RnGmhOk_XQeJtbqQ.

14. Antunes JM, Daher DV, Giaretta VM, Ferrari MF, Posso MB. Hydrotherapy and crenotherapy in the treatment of pain: integrative review. BrJP. 2019 abr-jun; 2(2):187-98

15. Mallen-Perez $\mathrm{L}$, et al. Use of hydrotherapy during labour: Pain, analgesia and neonatal safety. EnfermClin. 2018; 28(5):309-315.

16. Santos, et al. Relato de experiência no contexto da educação em saúde o cuidado materno-infantil. Rev. Enferm. UFPE online. 2015; 9(5):8478-8.

17. Sousa, et al. Ações de enfermagem na educação em saúde do pré-natal: relato de experiência. Rev. Interd. Enc. das Ciências. 2018; 1(3):348-361. 18. Campagnoli M, Silva CPD, Resende RCP. Atendimento de pré-natal na estratégia saúde da família: a singularidade da assistência de enfermagem. Nursing (Säo Paulo). 2019; 22(251): 2915-2920.

19. Pinheiro DGM, Scabar TG, Maeda ST, Fracolli LA, Pelicioni MCF, Chiesa AM. Health promotion competencies: challenges of formation Saude soc. 2015 jan.l mar.; 24(1).

20. Soares AM, Pereira MAS, Canavarro JMAP. Promoção da saúde nas Instituições de Ensino Superior Portuguesas: Reflexões e Desafios. revista portuguesa de pedagogia. 2015; 49(2):115-137. 